\title{
In vivo, dual-modality imaging of mouse eyes: optical coherence tomography and photoacoustic microscopy within a single instrument
}

Bin Rao, Li Li, Konstantin Maslov, Lihong V. Wang

Bin Rao, Li Li, Konstantin Maslov, Lihong V. Wang, "In vivo, dual-modality imaging of mouse eyes: optical coherence tomography and photoacoustic microscopy within a single instrument," Proc. SPIE 7550, Ophthalmic Technologies XX, 75501Q (2 March 2010); doi: 10.1117/12.843041 


\title{
In vivo, dual-modality imaging of mouse eyes: optical coherence tomography and photoacoustic microscopy within a single instrument
}

\author{
Bin Rao, Li Li, Konstantin Maslov and Lihong V. Wang \\ Optical Imaging Laboratory, Department of Biomedical Engineering, Washington University in St. \\ Louis, St. Louis, MO 63130
}

\begin{abstract}
Optical-resolution photoacoustic microscopy (OR-PAM) provides superb optical absorption contrast for red blood cells (RBCs), which makes it ideal for in vivo microvasculature imaging. In comparison, optical coherence tomography (OCT), widely used for tissue microstructure imaging, provides high optical scattering contrast. The two contrast mechanisms are highly complementary. In this work, we combined OR-PAM and OCT into a single, dual-modality imaging instrument for in vivo mouse eye imaging. We demonstrated in vivo dual-modality imaging of the anterior segment of mouse eyes with laser pulse energy within the ANSI laser safety standard.
\end{abstract}

Keywords: optical coherence tomography, photoacoustic microscopy, anterior segment of eye, iris imaging

\section{INTRODUCTION}

Optical coherence tomography (OCT) [1] has become the mainstream imaging technology in ophthalmology. OCT has high sensitivity in imaging depth-resolved subsurface tissue structures using coherence gating and coherence amplification. Contrast mechanisms such as Doppler contrast, polarization contrast, spectroscopic contrast, and speckle contrast are important in many OCT applications. The above contrast mechanisms are based on optical scattering properties. In ophthalmology, OCT technology does not satisfy all clinical needs. Only limited functional imaging information is provided by the Doppler OCT. Invasive fluorescein angiography (FA) and indocyanine green angiography (ICGA) are still widely used in eye clinics. In vivo, label-free imaging technologies with endogenous contrast are always desirable for replacing the invasive imaging technologies. The recent development of opticalresolution photoacoustic microscopy (OR-PAM) [2], which is based on detecting the optical absorption contrast mechanism, introduces a novel member into the family of optical diagnosing tomography. OR-PAM, along with darkfield acoustic-resolution photoacoustic microscopy (AR-PAM), photoacoustic computed tomography and thermoacoustic tomography, promises to have a substantial impact on biomedical applications in the near future $[3,4]$. In fact, PAM and OCT are complementary technologies since they provide different contrasts. In this work, we combined OR-PAM and OCT within a single, dual-modality imaging instrument for in vivo mouse eye imaging. We demonstrated in vivo dual-modality (OCT and OR-PAM) imaging of the anterior segment in mouse eyes with laser pulse energy within the ANSI laser safety standard.

\section{MATERIALS AND METHODS}

Schematic dual-modality OCT/PAM imaging system setup

The dual-modality OCT/PAM imaging system setup is shown in Figure 1. The dual-modality imaging probe, which is shown as a green dashed box in Figure 1, will be discussed in next section. The whole system includes a traditional Fourier-domain OCT system [5], a $532 \mathrm{~nm}$ pulsed laser, one AO card (NI-6713), one A/D card (NI-5124), and the dualmodality imaging probe. The AO card was used to generate trigger signals to fire laser pulses and move the 1D stage. The PAM signal detected by the ultrasonic transducer was detected by the NI-5124 card. The pulsed laser has a pulse duration of $2 \mathrm{~ns}$. Its pulse repetition rate was set as 5000. The pulse energy was set as $60 \mathrm{~nJ}$ for in vivo imaging.

The Fourier-domain OCT system includes a broadband light source (Superlum D890, $150 \mathrm{~nm} 3 \mathrm{~dB}$ bandwidth, center wavelength $890 \mathrm{~nm}$ ), an optical isolator, a 20/80 split-ratio 2 by 2 fiber coupler, a reference arm, a sample arm, and a spectrometer in the detection arm. The customized spectrometer was used to detect low back-scattered light from the sample. The CCD line-scan camera has 2048 pixels. The pixel size is $14 \mu \mathrm{m} \times 14 \mu \mathrm{m}$. The maximum frame rate of

Ophthalmic Technologies XX, edited by Fabrice Manns, Per G. Söderberg, Arthur Ho, Proc. of SPIE Vol. 7550, 75501Q · C 2010 SPIE $\cdot$ CCC code: 1605-7422/10/\$18 - doi: 10.1117/12.843041 
CCD-line scan camera is 29,000 . OCT system's signal-to-noise ratio (SNR) was measured as $100 \mathrm{~dB}$ with $750 \mu \mathrm{w}$ sample arm power and $50 \mu \mathrm{s} \mathrm{CCD} \mathrm{A-line} \mathrm{integration} \mathrm{time.} \mathrm{Comparing} \mathrm{to} \mathrm{the} \mathrm{SNR} \mathrm{measured} \mathrm{at} \mathrm{the} \mathrm{imaging} \mathrm{depth} \mathrm{of} 50$ $\mu \mathrm{m}$, a $9 \mathrm{~dB}$ of SNR roll-off was observed at the imaging depth of $2 \mathrm{~mm}$. The dual-modality imaging system was operating at 5000 Alines per second, which was limited by the pulse repetition rate of the laser.

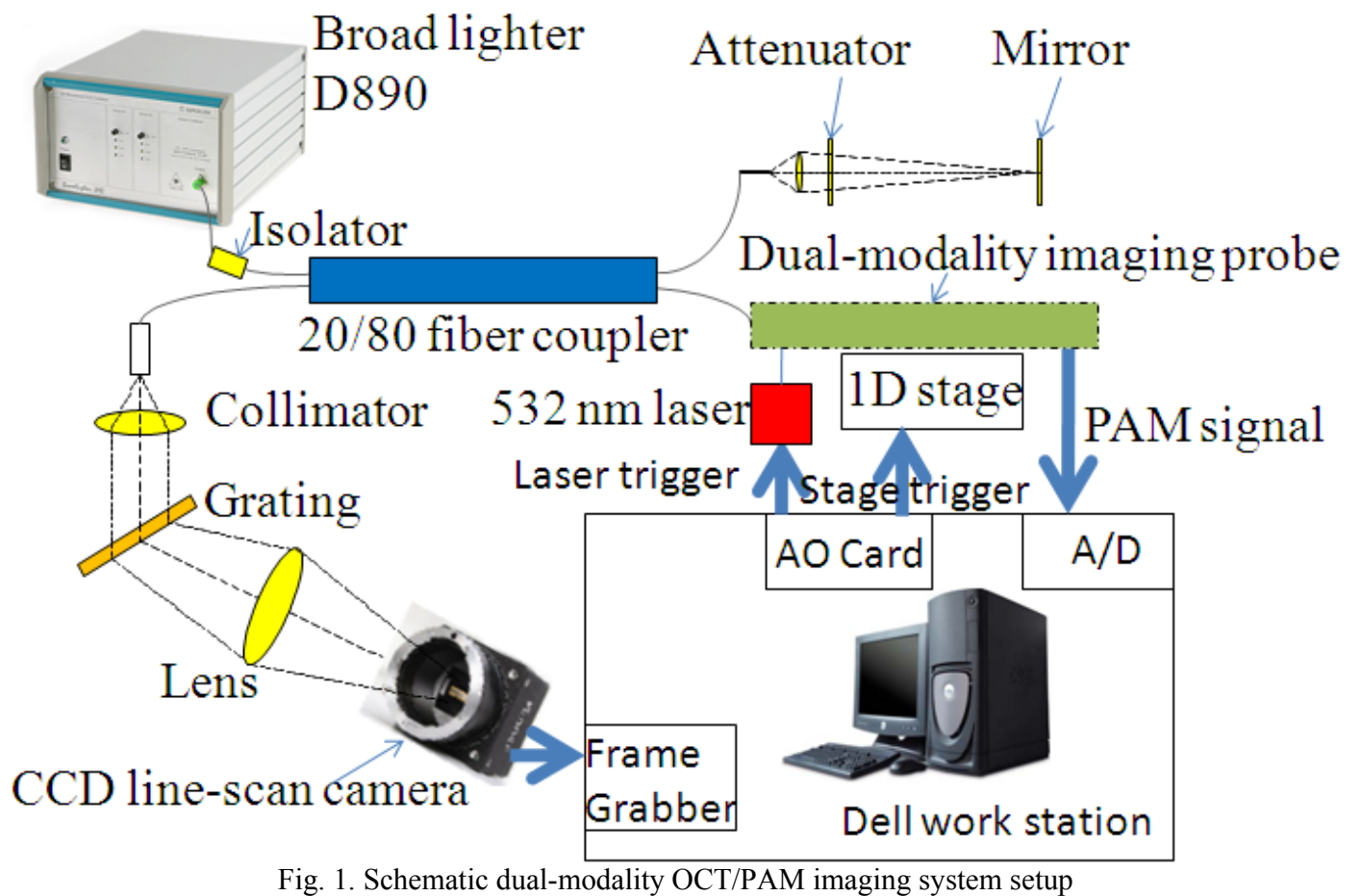

Schematic setup of the OCT/PAM dual-modality imaging probe for ocular anterior segment imaging [6, 7]

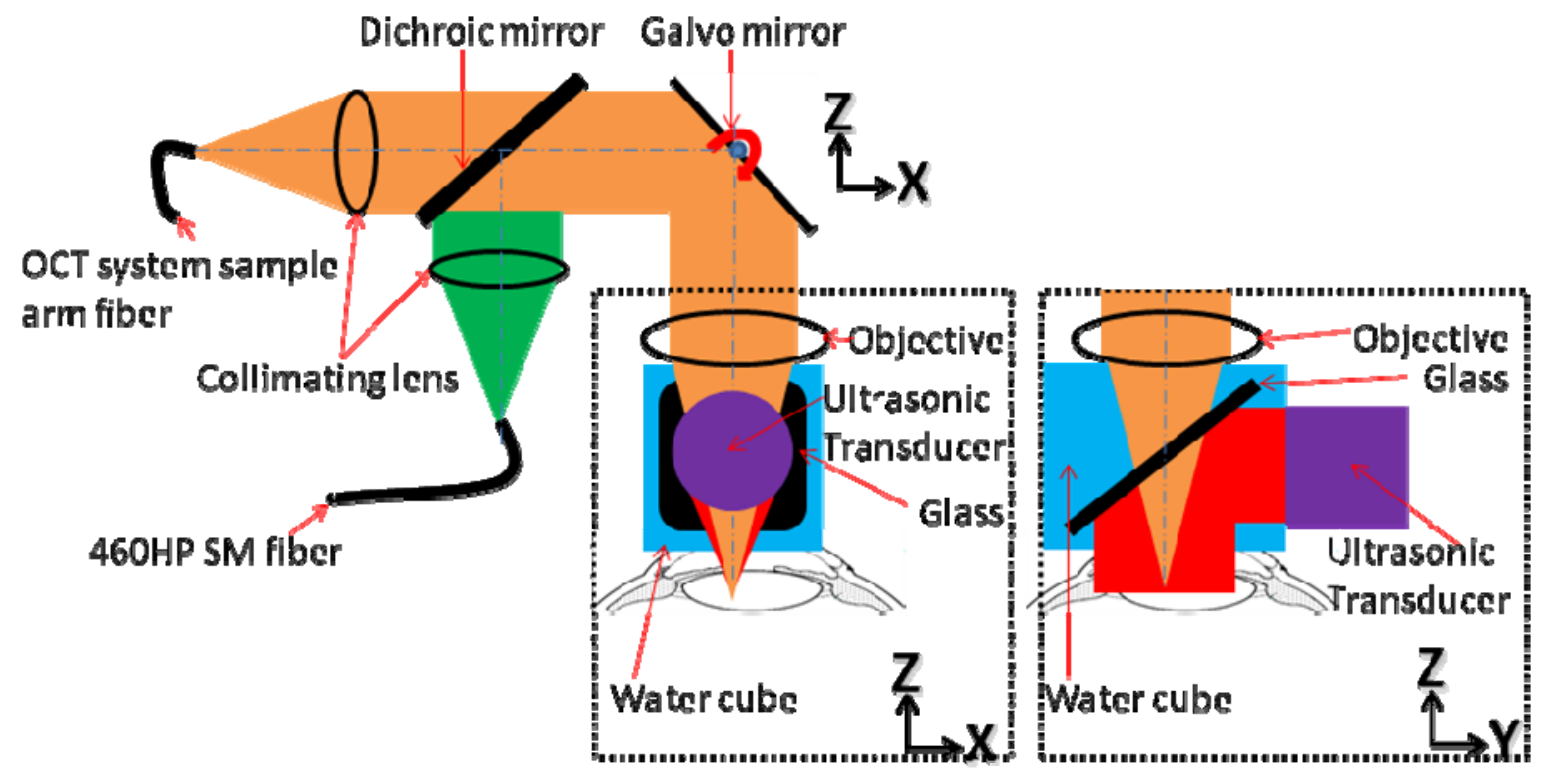

Fig. 2. Schematic setup of the OCT/PAM dual-modality imaging probe 
Figure 2 shows the schematic setup of the dual-modality OCT/PAM imaging probe. The sample arm light from the OCT system was connected to the dual-modality probe through a HI-780 single mode fiber. The $532 \mathrm{~nm}$ pulsed laser was connected to the probe with a $460 \mathrm{HP}$ single mode fiber. A diachronic mirror was used to combine the collimated OCT sample arm light and $532 \mathrm{~nm}$ PAM excitation laser. A Galvo mirror, which was located at the front focus of an objective (NA 0.1) was used to deflect the laser beams. A water cube comprising two isosceles right-angle water prisms and a glass plate between them was employed for light wave delivery and acoustic wave coupling. After passing through the water cube, the laser beam was focused at least $200 \mu \mathrm{m}$ below the tissue surface. Upon the absorption of the laser pulse by the tissue, photoacoustic waves were generated. Thereafter, it was reflected by the glass plate and detected by the ultrasonic transducer. The ultrasonic transducer (GE, $25 \mathrm{MHz}$ bandwidth), having a line focus along the optical scan axis, collected photoacoustic signals from the fast optical scan axis (X axis) without being moved mechanically. The whole probe was attached to a one-dimensional mechanical stage, which scanned perpendicularly to the fast optical scan axis. The OCT A-line image and PAM Aline image were acquired sequentially in time.

\section{Schematic OCT/PAM system software architecture}

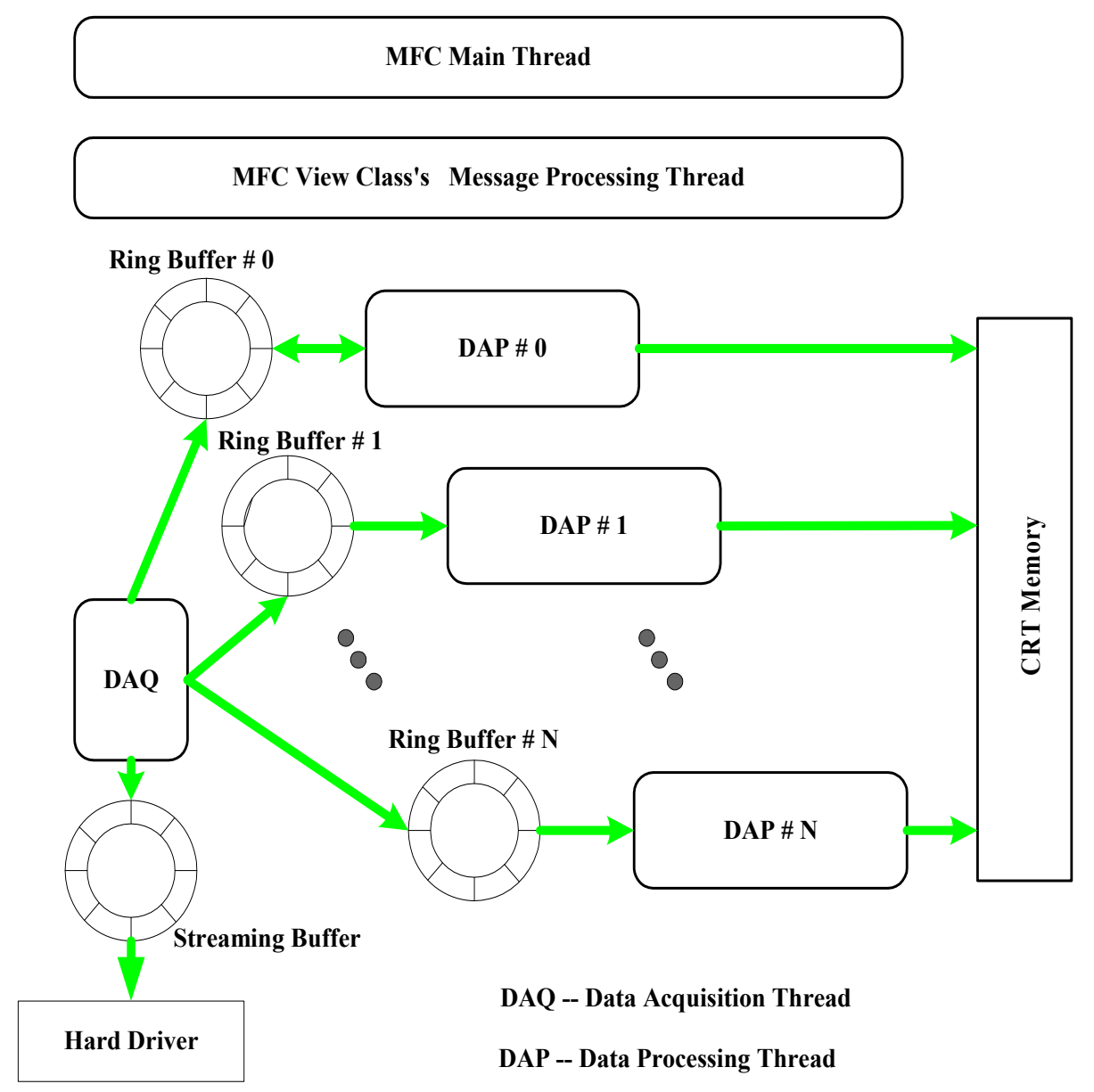

Fig. 3. Schematic OCT/PAM system software architecture

Figure 3 shows the schematic OCT/PAM dual-modality imaging system software architecture. The software was written in Visual $\mathrm{C}++$. Multiple threads were used for real time data acquisition (DAQ), data processing (DAP) and image presentation tasks. The DAQ thread acquired data from both the frame grabber and the NI-5124 card. Raw data was sent to the streaming buffer for documentation. Data frames were sent to different DAP threads for real time data processing and data presentation. The number of DAP threads were scalable in software. 


\section{Imaging procedures}

All experimental animal procedures were carried out in conformity with the laboratory animal protocol approved by the School of Medicine Animal Studies Committee of Washington University in St. Louis. Nude mice (Hsd, Athymic Nude-Foxn $1^{\mathrm{NU}}$, Harlan Co.) were anesthetized by intraperitoneal (IP) injection of a cocktail containing Ketamine (100 $\mathrm{mg} / \mathrm{kg}$ ) and Xylazine $(10 \mathrm{mg} / \mathrm{kg}$ ). A custom-made animal holder was used to restrain the animal. A breathing device (E$\mathrm{Z}$ Anesthesia, Euthanex Corp.) was employed to maintain the anesthesia status after the IP injection. The mouse was placed under a water tank to maximize the coupled acoustic wave for best ultrasonic detection.

In order to acquire a 3D image, an imaging area of $4 \mathrm{~mm} \times 4 \mathrm{~mm}$ was scanned. The step size in both lateral directions was $5 \mu \mathrm{m}$. The size of 3D PAM data set was $800 \times 800 \times 200$ while the size of 3D OCT data set was $800 \times 800 \times 2048$.

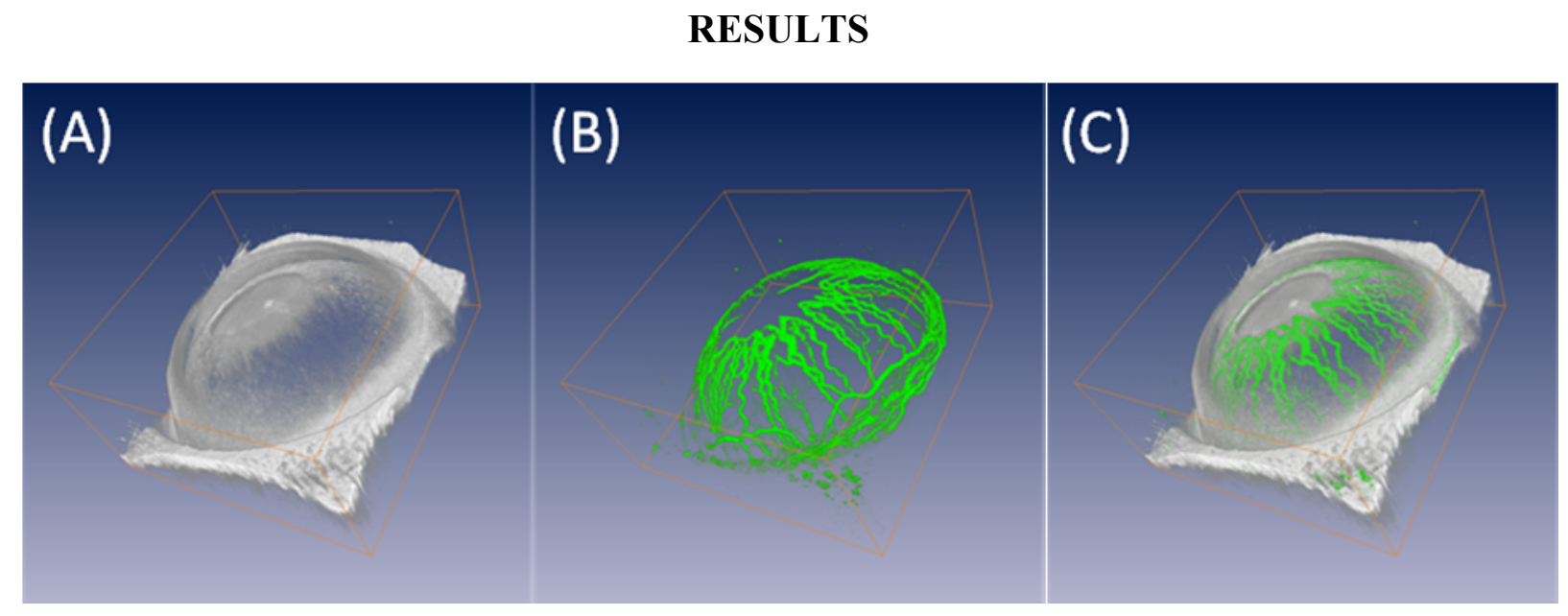

Fig 3. In vivo, label-free mouse ocular anterior segment imaging in (A) OCT mode (B) PAM mode and (C) dual-modality mode

Figure 3 shows the in vivo, label-free ocular anterior segment imaging results. Figure 3A showed only a 3D OCT image of the anterior segment of the mouse eye. Figure 3B showed only a 3D PAM image of the anterior segment of the mouse eye. Combing the contrast in Fig 3A and Fig 3B, Figure 3C showed a dual-modality 3D OCT/PAM image of the anterior segment of the mouse eye. As shown in Figure 3B, PAM has superb contrast to red blood cells in vasculature while OCT has good optical scattering contrast in documenting ocular anterior segment structure. The combination of contrast mechanisms of OCT and PAM may be helpful in diagnosing anterior segment eye diseases in the future.

\section{LASER SAFETY CONSIDERATIONS}

The major laser safety concern for the anterior segment of eye imaging is the safety of anterior segment tissue instead of retinal tissue [6]. We assume the laser focus is $200 \mu \mathrm{m}$ below the surface tissue. The surface illumination area (NA = $0.1)$ is $30 \mu \mathrm{m}$. We scanned $4 \mathrm{~mm}$ using 800 points. The step size was $5 \mu \mathrm{m}$. Thus the repetitive pulse number on the illumination area was 6 . The repetitive pulse limit can be calculated as the following:

$$
\mathrm{MPE}_{\mathrm{RP}}=\mathrm{n}^{-0.25} \times \mathrm{MPE}_{\mathrm{SP}}=6^{-0.25} \times 20 \mathrm{~mJ} / \mathrm{cm}^{2}=12.78 \mathrm{~mJ} / \mathrm{cm}^{2}
$$

After multiplying the $\mathrm{MPE}_{\mathrm{RP}}$ with surface illumination area, the maximum permissible pulse energy is calculated as 90 $\mathrm{nJ}$ per pulse. Thus our pulse energy of $60 \mathrm{~nJ}$ per pulse is within safety limit.

\section{CONCLUSIONS}

In vivo, real time imaging and visualization of both microvasculature and microstructure simultaneously are desirable for many biomedical applications. We demonstrated in vivo dual-modality (OCT and OR-PAM) imaging of the anterior segment in a mouse eye with laser pulse energy within ANSI laser safety standard. The endogenous contrasts make this instrument safer than the conventional fluorescence dye based imaging devices in diagnosing anterior segment eye diseases. 


\section{ACKNOWLEDGEMENT}

The OCT/PAM dual-modality system and mouse ocular anterior segment imaging demonstration were contributed in equal measure by Bin Rao and $\mathrm{Li} \mathrm{Li}$. The research was supported by the National Institutes of Health grants R01 EB000712, EB000712A2S1, R01 EB00071207S2, R01 EB008085, R01 CA113453901, U54 CA136398, and 5P60 DK02057933. L.W. has a financial interest in Microphotoacoustics, Inc. and Endra, Inc., which, however, did not support this work.

\section{REFERENCES}

[1] D. Huang, E. A. Swanson, C. P. Lin, J. S. Schuman, W. G. Stinson, W. Chang, M. R. Hee, T. Flotte, K. Gregory, C. A. Puliafito, and J. G. Fujimoto, "Optical coherence tomography," Science 254, 1178-1181 (1991).

[2] K. Maslov, H. F. Zhang, S. Hu, and L. V. Wang, "Optical-resolution photoacoustic microscopy for in vivo imaging of single capillaries," Optics Letters 33, 929-931 (2008).

[3] L. V. Wang, "Prospects of photoacoustic tomography," Medical Physics 35 (12), 5758-5767 (Dec. 2008).

[4] Lihong Wang, "Multiscale photoacoustic microscopy and computed tomography," Nature Photon. 3, 503509(2009).

[5] Bin Rao, Lingfeng Yu, Huihua Kenny Chiang, Leandro C. Zacharias, Ronald M. Kurtz, Baruch D. Kuppermann and Zhongping Chen, "Imaging pulsatile retinal blood flow in human eye," J. Bio. Opt. 13(4) 040505(2008).

[6] Bin Rao, Li Li, Konstantin Maslov, and Lihong V. Wang, "Hybrid-scan optical-resolution photoacoustic microscopy," (to be submitted to OL).

[7] L. Li, B. Rao, K. Maslov, and L. V. Wang, "In vivo imaging of cutaneous microcirculation by fast-scanning reflection-mode integrated photoacoustic and optical coherence microscopy," to be submitted to Optical Letters.

[8] Song Hu, Bin Rao, Konstantin Maslov, and Lihong V. Wang, "Label-free photoacoustic ophthalmic angiography," Opt. Lett. 35, 1-3 (2010).

[9] Laser Institute of America, American National Standard for Safe Use of Lasers ANSI Z136.1-2000 (American National Standards Institute, Inc., 2000). 